\title{
Analysis of a passive control of a chain under wide-band random excitation by nonlinear energy sinks using generalized orthogonal decompositions
}

\author{
S. Bellizzi ${ }^{1, a}$ and R. Sampaio ${ }^{2}$ \\ 1 LMA, CNRS, UPR 7051, Centrale Marseille, Aix-Marseille Univ, F-13420 Marseille Cedex 20, France. \\ 2 PUC-Rio, Dept of Mechanical Engineering, Rua Marquês de São Vicente 225, 22453-900 Rio de Janeiro, Brazil.
}

\begin{abstract}
The objective of this paper is to show how the generalized orthogonal decomposition named smooth decomposition can be used to analyze the energy pumping phenomenon in the context of vibration reduction under wide-band random excitation.
\end{abstract}

\section{Introduction}

The Targeted Energy Transfer (TET) approach represents a concept in which a strongly purely nonlinear, passive, local attachment, the Nonlinear Energy Sink (NES), is employed to reduce the vibrations of the primary system to which it is attached. The NES can passively absorb and locally dissipate energy from the primary structure. The energy interactions occur due to internal resonances making possible irreversible nonlinear energy transfers from the primary system to the NES component. The purely nonlinearity of the NES enables it to resonate with any modes of the primary structure. A description of the TET can be found in [1]. The TET concept was principally analyzed in the literature in a deterministic framework. In this study, wide-band random excitations will be considered.

Generalized orthogonal decompositions provide a powerful tool for random vibrations analysis. The most popular orthogonal decomposition is the Karhunen-Loève Decomposition (KLD). Recently, a modified decomposition, that is not orthogonal in the euclidean sense, named Smooth Decomposition (SD) has been proposed[2][3][4]. The SD can be view as a projection of an ensemble of spatially distributed data such that the vector directions of the projection not only keep the maximum possible variance but also the motions resulting along the vector directions are as smooth as possible in time. The vector directions (or structures or smooth modes) are defined as the eigenvectors of an eigenproblem defined from the covariance matrices of the random field and of the associated time derivative. It was shown that the SD is an interesting tool to random analysis. The parameters of the SD can be interpreted in terms of normal modes and resonance frequencies given access to an modal analysis of the random problem. With these properties, the SD analysis gives a dual interpretation. The modes given by the SD can be ordered through frequency, as classical modal analysis does, and through energy levels, as KLD does. This makes the SD a powerful tool to analyze nonlinear systems in a way similar to modal analysis of linear systems or in a way similar to KLD.

In this paper, the SD will be used to analyze a chain of $M$ strongly coupled linear oscillators (the primary system)

\footnotetext{
a e-mail: bellizzi@lma.cnrs-mrs.fr
}

with a strongly nonlinear end-attachment (the NES). This system was studied in [5] considering impulsive excitation. We propose here to analyze the targeted energy transfer when the excitation is white-noise random process. This kind of excitation differs significantly from the deterministic case but in terms of frequency contents, a white-noise excitation is similar to an impulsive excitation in the deterministic case. It permits to analyze the system without privileging a frequency band.

\section{The system under study}

\subsection{Description of the system}

The system is composed of a chain of $M$ strongly coupled linear oscillators with spring $\left(k_{c}\right)$ (named the linear chain or the primary system) with a strongly nonlinear endattachment (the NES). Each mass of the linear chain is connected to the ground by a linear spring $\left(k_{g}\right)$ and a linear dashpot $\left(\lambda_{g}\right)$. The equations of motion are given by

$$
\begin{aligned}
m_{a} \ddot{v}+\lambda_{a}\left(\dot{v}-\dot{u}_{1}\right)+k_{a}\left(v-u_{1}\right)+C_{a}\left(v-u_{1}\right)^{3} & =0, \\
\ddot{u}_{1}+\lambda_{g} \dot{u}_{1}+k_{g} u_{1}-\lambda_{a}\left(\dot{v}-\dot{u}_{1}\right)-k_{a}\left(v-u_{1}\right) & \\
-C_{a}\left(v-u_{1}\right)^{3}+k_{c}\left(u_{1}-u_{2}\right) & =0, \\
\ddot{u}_{m}+\lambda_{g} \dot{u}_{m}+k_{g} u_{m}+k_{c}\left(2 u_{m}-u_{m-1}-u_{m+1}\right) & =0, \\
\ddot{u}_{M}+\lambda_{g} \dot{u}_{M}+\left(k_{g}+k_{c}\right) u_{M}+k_{c}\left(u_{M}-u_{M-1}\right) & =f(t)
\end{aligned}
$$

with $m=1, \cdots, M-1$ and where $v$ (respectively $u_{m}$ ) denotes the displacement of the NES (respectively the $m$ th mass of the linear chain). It is assumed that the primary system possesses a weak viscous damping ( $\lambda_{g}$ is small). The NES is constituted of a mass $\left(m_{a}\right)$, a linear damper $\left(\lambda_{a}\right)$ and a spring including a linear part $\left(k_{a}\right)$ and a cubic part $\left(C_{a}\right) . m_{a}$ is assumed to be small compared to the total mass of the linear chain and the linear spring is assumed to be small compared to cubic spring. This system was considered in [5] under impulsive excitation.

We assume that the excitation is of the form

$$
f(t)=s_{0} W(t)
$$

where $\{W(t), t \in \mathbb{R}\}$ is a gaussian white-noise scalar process with intensity one and $s_{0}$ denotes the excitation level. 


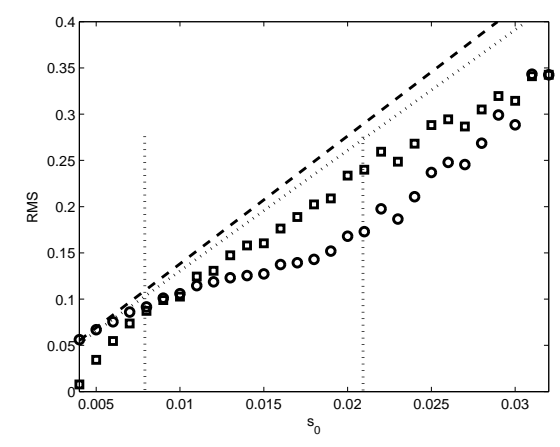

Fig. 1. $R M S_{\text {chain }}$ for the system with the NES (circle markers), with only the linear part of the NES (dotted line), without NES (dashed line) and $R M S_{N E S}$ (square markers) versus level excitation $s_{0}$.

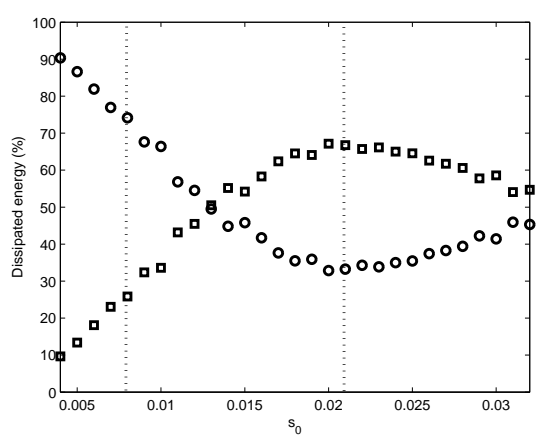

Fig. 2. Percentage of energy dissipated by the linear chain (circle markers) and by the NES (square markers) versus level excitation $s_{0}$.

\subsection{Passive capacity of vibration reduction}

We limit the discussion to some numerical evidences showing that the NES is able to absorb vibrational energy of the linear chain.

The stationary responses of the system (1)-(4) were investigated using the following numerical parameter values: $M=9, \lambda_{g}=0.001, k_{g}=1, k_{c}=1, m_{a}=0.05, \lambda_{g}=0.001$, $k_{a}=0.0001, C_{a}=1$. The excitation levels $s_{0}$ was used as the parameter of analysis with $s_{0} \in[0.004,0.032]$.

The Monte-Carlo method was used to estimate the stationary responses of the system under random excitation. For a given excitation level, the response time history (displacement and velocity) was obtained from an time history of excitation $W(t)$ by solving Eqs. (1)-(4) over the time interval $\left[0, t_{f}\right]$ numerically using the Newmark method. Zero initial displacement and velocity were assumed. The time-discretization parameter value was chosen equal to $\Delta t=0.143$ s (i.e. $\left.f_{e}=7 \mathrm{~Hz}\right)$ and 524286 instants $\left(t_{f}=\right.$ $74942 \mathrm{~s})$ were simulated. The time histories of $W(t)$ (a gaussian white-noise scalar process with intensity one) were generated using a FFT method[6]. The last-half points of the displacement and velocity time histories were used to approximate the second order moments (as the time averages).

The evolution of the RMS values of the NES displacement $\left(R M S_{N E S}=\sqrt{\mathbb{E}\left(v^{2}(t)\right)}\right)$ and the RMS values of the primary system $\left(R M S_{\text {chain }}=\sqrt{\sum_{m=1}^{M} \mathbb{E}\left(u_{m}^{2}(t)\right)}\right)$ versus $s_{0}$ are displayed Fig. 1.
For small $s_{0}$, significant vibrations occur only on the linear chain so the behavior of the system is close to the behavior of the linear configurations. When $s_{0}$ increases, the vibrations of the NES increase and simultaneously the vibrations of the linear chain are significantly reduced compared to the two linear configurations. Particularly interesting is that a zone (defined by $0.008 \leq s_{0} \leq 0.021$ ) appears where $R M S_{\text {chain }}$ does not significantly increase with $s_{0}$. This zone will be named "effective" zone. Finally for large values of $s_{0}$, the vibrations of the linear chain again increase linearly.

An important measure to evaluate the performance of NES is given by the energy dissipated by the NES. The energy dissipated by the linear chain and by the NES is respectively given by

$$
E_{\text {chain }}^{d}=\lambda_{0} \sum_{m=1}^{M} \mathbb{E}\left(\dot{u}_{m}^{2}(t)\right) \text { and } E_{N E S}^{d}=\lambda_{N E S} \mathbb{E}\left(\left(\dot{v}(t)-\dot{u}_{1}(t)\right)^{2}\right)
$$

The percentages of energy dissipated by the linear chain $\left(E_{\text {chain }}^{d} /\left(E_{\text {chain }}^{d}+E_{N E S}^{d}\right)\right)$ and by the NES $\left(E_{N E S}^{d} /\left(E_{\text {chain }}^{d}+\right.\right.$ $\left.\left.E_{N E S}^{d}\right)\right)$ are reported versus the excitation level in Fig. 2.

For small $s_{0}$, the energy is mainly dissipated by the linear chain. When $s_{0}$ increases, the percentage of energy dissipated by the linear chain decreases whereas the percentage of energy dissipated by the NES increases. The optimal performance of the NES is obtained for $s_{0} \approx 0.021$ where $70 \%$ of energy is dissipated by the nonlinear endattachment. This value corresponds to the upper bound of the "effective" zone. Finally for large values of $s_{0}$, the percentage of energy dissipated by the NES decreases whereas the percentage of energy dissipated by the linear chain turns to increase and becomes greater than the percentage of energy dissipated by the NES. The energy pumping phenomena vanishes.

These results indicate that the NES modifies significantly the dynamic of the linear chain. In reference of the excitation level, three behaviors can be observed. For small values of $s_{0}$ no coupling appears between the linear chain and the NES. When a specific threshold is exceeded, the vibrations of the NES become large whereas the vibrations of the linear chain are significantly reduced compared to the linear cases. This is the energy pumping phase characterized by a transfer of energy from the primary system to the NES. This behavior characterizes the "effective" zone. Finally, the energy pumping phenomenon vanishes below a certain level of excitation.

The performance of the NES can also be analyzed in the frequency domain using the PSD function (not shown here).

\section{Smooth decomposition}

Let $\{\mathbf{U}(t), t \in \mathbb{R}\}$ be a $\mathbb{R}^{n}$-valued second-order stationary random process with zero mean indexed by $\mathbb{R}$. We assume that $\{\mathbf{U}(t), t \in \mathbb{R}\}$ has a time-derivative process $\{\dot{\mathbf{U}}(t), t \in$ $\mathbb{R}\}$ which is also a second-order stationary process. The covariance matrices of $\{\mathbf{U}(t), t \in \mathbb{R}\}$ and $\{\dot{\mathbf{U}}(t), t \in \mathbb{R}\}$ are denoted $\mathbf{R}_{\mathbf{U}}=\mathbb{E}\left(\mathbf{U}(t)^{T} \mathbf{U}(t)\right)$ and $\mathbf{R}_{\mathbf{U}}=\mathbb{E}\left(\dot{\mathbf{U}}(t)^{T} \dot{\mathbf{U}}(t)\right)$ respectively. 
The SD of $\{\mathbf{U}(t), t \in \mathbb{R}\}$ is defined by the series in the separated-variables form

$$
\mathbf{U}(t)=\sum_{k=1}^{n} a_{k}^{S}(t) \boldsymbol{\Phi}_{k}^{S}
$$

where the Smooth Components (SCs) are defined by

$$
a_{k}^{S}(t)=\frac{\boldsymbol{\Phi}_{k}^{S^{T}} \mathbf{R}_{\mathbf{U}} \mathbf{U}(t)}{\boldsymbol{\Phi}_{k}^{S^{T}} \mathbf{R}_{\mathbf{U}} \boldsymbol{\Phi}_{k}^{S}}=\frac{\boldsymbol{\Phi}_{k}^{S^{T}} \mathbf{R}_{\mathbf{U}} \mathbf{U}(t)}{\boldsymbol{\Phi}_{k}^{S^{T}} \mathbf{R}_{\mathbf{U}} \Phi_{k}^{S}}
$$

and the Smooth Modes (SMs) $\boldsymbol{\Phi}_{k}^{S}$ are characterized by the optimization problem

$$
\max _{\boldsymbol{\Phi} \in \mathbb{R}^{n}} J_{S D}(\boldsymbol{\Phi}) \text { with } J_{S D}(\boldsymbol{\Phi})=\frac{\left.\mathbb{E}(<\mathbf{U}(t), \boldsymbol{\Phi})>^{2}\right)}{\mathbb{E}\left(<\dot{\mathbf{U}}(t), \boldsymbol{\Phi}>^{2}\right)}=\frac{\boldsymbol{\Phi}^{T} \mathbf{R}_{\mathbf{U}} \boldsymbol{\Phi}}{\boldsymbol{\Phi}^{T} \mathbf{R}_{\mathbf{U}} \boldsymbol{\Phi}}
$$

and solved the eigenproblem

$$
\mathbf{R}_{\mathbf{U}} \boldsymbol{\Phi}_{k}^{S}=\mu_{k}^{S} \mathbf{R}_{\mathbf{U}} \boldsymbol{\Phi}_{k}^{S} .
$$

Based on the properties of the matrices $\mathbf{R}_{\mathbf{U}}$ and $\mathbf{R}_{\mathbf{U}}$, the SMs $\left(\boldsymbol{\Phi}_{1}^{S}, \boldsymbol{\Phi}_{2}^{S}, \cdots, \boldsymbol{\Phi}_{n}^{S}\right)$ constitutes a $\mathbf{R}_{\mathbf{U}}$-orthogonal and $\mathbf{R}_{\mathbf{U}^{-}}$-orthogonal basis of $\mathbb{R}^{n}$ and all the eigenvalues named Smooth Values (SVs) are greater than zero. Notice that the following ordering, $\mu_{1}^{S} \geq \mu_{2}^{S} \geq \cdots \geq \mu_{n}^{S}>0$, will be used in the sequel.

All the properties of the SD are reported in [3]. We will just recalled here the physical interpretation of the parameters of the SD. We assume that $\mathbf{R}_{\mathbf{U}}$ and $\mathbf{R}_{\mathbf{U}}$ are the covariance matrices of the steady state solution of a discrete linear mechanical system under zero-mean white-noise random excitation. If the damping is proportional and if the modal-excitation terms are uncorrelated then the following results hold:

- the SMs are related to the normal modes by

$$
\boldsymbol{\Phi}^{S}=\boldsymbol{\Phi}^{L^{-T}}
$$

where $\boldsymbol{\Phi}^{S}=\left[\boldsymbol{\Phi}_{1}^{S} \boldsymbol{\Phi}_{2}^{S} \ldots \boldsymbol{\Phi}_{n}^{S}\right]$ and $\boldsymbol{\Phi}^{L}=\left[\boldsymbol{\Phi}_{1}^{L} \boldsymbol{\Phi}_{2}^{L} \cdots \boldsymbol{\Phi}_{n}^{L}\right]$ denotes the modal matrix associated to the undamped linear system;

- the SVs are related to the natural resonance frequencies by

$$
\boldsymbol{\mu}^{S}=\left(\boldsymbol{\Omega}^{\mathbf{2}}\right)^{-1}
$$

where $\boldsymbol{\mu}^{S}=\operatorname{diag}\left(\mu_{k}^{S}\right)$ and $\boldsymbol{\Omega}^{\mathbf{2}}=\operatorname{diag}\left(\omega_{k}^{2}\right)$ with $\omega_{k}$ denotes the natural resonance frequencies associated to the undamped linear system.

As it will be shown hereafter, the relations (9) and (10) can be used to perform modal analysis from SD.

\section{Smooth decomposition analysis}

The SD approach gives access to the smooth parameters but also to the classical modal parameters. We will focus here on these characteristics. The smooth parameters were obtained solving the eigenproblem (8) using the covariance matrices $\mathbf{R}_{\mathbf{U}}$ and $\mathbf{R}_{\dot{U}}$ of the response of the system (1)-(4) estimated from the numerical simulations (see Section 2.1). Same data have been used as in Section 2.2.

The resonance frequencies estimated from SVs (with Eq. (10)) are shown Fig. 3 whereas the percentage of energy captured by the SMs are plotted Fig. 4 .

The following observations can be made:

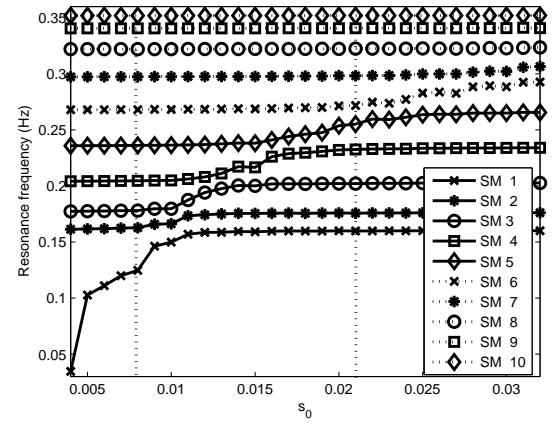

Fig. 3. SD of the system with NES: resonance frequencies estimated from SD versus level excitation $s_{0}$.

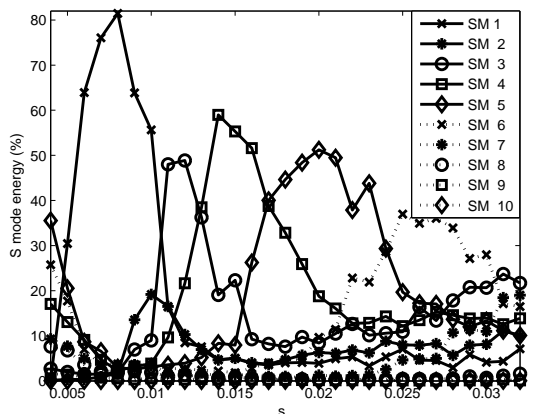

Fig. 4. SD of the system with NES: percentage of energy captured by the SMs versus excitation level $s_{0}$ (right).

- For $s_{0}=0.004$, the ten resonance frequencies estimated from the SVs are related to the natural resonance frequencies of the underlying linear system (i.e. $C_{a}=0$ ) (see Fig. 3). The smaller resonance frequency $(\approx 0.04 \mathrm{~Hz})$ is greater than the natural frequency of the linear part of the NES, the nine remaining frequencies are equal to the natural frequencies of the linear chain.

- For $s_{0}$ between 0.04 and 0.08 , the first resonance frequency estimated from the SVs rapidly increases up to the frequency value $0.16 \mathrm{~Hz}$ which corresponds to the natural resonance frequency of the first mode of the linear chain whereas all the nine remaining resonance frequencies estimated from the SVs remain constant. For this excitation level band, the energy is captured by the first SM. The maximum value of the percentage of captured energy $(\approx 82 \%)$ is obtained for $s_{0} \approx 0.008$.

- Around $s_{0}=0.01$, the second resonance frequency $(0.16 \mathrm{~Hz})$ estimated from the SVs (i.e. the resonance frequency of the first normal mode of the linear chain) begins to increase whereas the first resonance frequency estimated from the SVs becomes asymptotic (with respect the excitation level) to $0.16 \mathrm{~Hz}$. For this excitation level, the energy is concentrated on the second SM. At this excitation level, this resonance interaction can be interpreted as a resonance capture.

- Increasing slightly $s_{0}$, the third resonance frequency $(0.175 \mathrm{~Hz})$ estimated from the SVs (i.e. the resonance frequency of of the second normal mode of the linear chain) begins to increase whereas the second resonance frequency estimated from the SVs (i.e. the resonance frequency of the first normal mode of the linear chain) becomes asymptotic (with respect the excitation level) to $0.175 \mathrm{~Hz}$. At this level, the energy becomes con- 

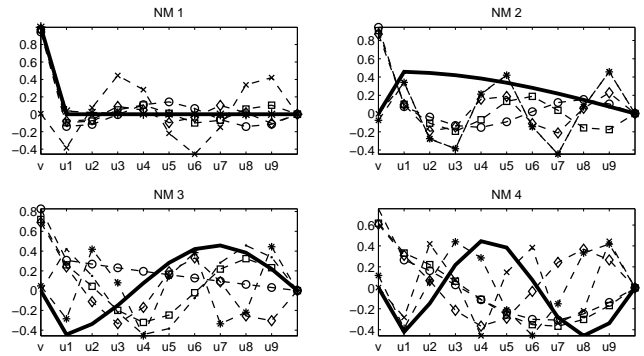

Fig. 5. SD of the system with NES: mode shapes of the first four normal modes estimated from the SMs ordering with respect to the modal energy for $s_{0}=0.004$ (cross markers), 0.008 (asterisk markers), 0.013 (circle markers), 0.019 (square markers) and 0.027 (diamond markers). The normal modes of the underlying linear system is also depicted (red line).

centrated on the third SM. The maximum value of the percentage of captured energy $(\approx 60 \%)$ is obtained for $s_{0} \approx 0.012$. At this excitation level, this resonance interaction can be interpreted as a resonance capture.

- Still increasing the level $s_{0}$, resonance interactions appear involving successively the higher resonance frequencies of the normal mode of the linear chain. This behavior can be interpreted as a resonance captures cascades. This behavior are related to the left shift of the resonant peak observed on the PSD of $\left(u_{i}\right)$ (not shown here).

Compared to a KLD analysis, more informations have been deduced from the SD analysis. In particular, the resonance capture phenomenon as well as the resonance captures cascades phenomenon have been revealed. These observations are very similar to that presented in [5] where impulsive excitations were used.

A complementary analysis can be derived from the SD approach ordering the SMs with respect to the energy captured by each SM (i.e. the energy of the S components) starting from the highest energy component to the lowest one. In Fig. 5, the mode shapes of the first four normal modes estimated from the SMs ordering with respect the energy of the SCs are displayed for five different excitation level values. We also reported the mode shape of the normal modes of the underlying linear system (i.e. $C_{N E S}=0$ ).

From Fig. 5, we can make the following observations:

- For $s_{0}=0.004$, the mode shapes of the first four energical dominant SMs coincide with the mode shapes of the first four dominant KL modes of the underlying linear system (i.e. $C_{N E S}=0$ ).

- For $s_{0} \geq 0.008$, the mode shapes of the first energical dominant normal modes are nearly identical of the first normal mode of the underlying linear system (i.e. $\left.C_{N E S}=0\right)$. This mode is spatially localized on the NES. The localization of the mode shape of the energical dominant SM on the NES for large excitation level is an indication of transfer of energy from the linear chain towards the NES.

These observations are very similar to that obtained with the KL analysis. They confirm the importance of the ordering of the SMs. Two ordering can be used for SMs, one, the $\mu$-ordering as used in Eqs. (9) and (10) is correlated to the classical ordering of the resonance frequencies, the other, the energy-ordering is correlated with the ordering used given by KLD.

\section{Conclusions}

In this paper, a random nonlinear system that presents energy pumping phenomenon is analyzed using SD approach. The system presents features very similar to the ones observed in the deterministic case when the system is impulsively forced although the tools of analysis are completely different. The energy pumping occurs for some excitation level, it is due to a localization phenomenon and resonance captures with any mode of the system (in resonance captures cascades). The results confirm the efficiency of the $\mathrm{SD}$. The smooth modes represent well how the energy is distributed in the system and clearly point out the localization phenomenon (as KLD does) and the resonance captures cascades (KLD does not). Contrary to KLD, it is also remarkable how the distribution of energy is related to the frequencies associated with the SD.

\section{Acknowledgments}

The authors gratefully acknowledge the financial support of CAPES and COFECUB (Grant number Ph 672/10).

\section{References}

1. Vakakis A., Gendelman O., Bergman L., McFarland D., Kerschen G., and Lee Y., Nonlinear Targeted Energy Transfer in Mechanical and Structural Systems I \& II. (Springer, 2008).

2. Chelidze D. and Zhou W., Smooth orthogonal decomposition-based vibration mode identification. Journal of Sound and Vibration, 292, (2006) 461-473.

3. Bellizzi S. and Sampaio R., Smooth Karhunen-Loève decomposition to analyze randomly vibrating systems. Journal of Sound and Vibration, 325, (2009) 491-498.

4. Bellizzi S. and Sampaio R., Analysis of nonstationary random processes using Smooth decomposition. Journal of Mechanics of Materials and Structures, 6(7-8), (2011) 1137-1152.

5. Vakakis A., L.I. Manevitch L., O. Gendelman O., and Bergmand L., Dynamics of linear discrete systems connected to local essentially non-linear attachments. Journal of Sound and Vibration, 264, (2003) 559-577.

6. Poirion F. and Soize C., Simulation numérique des champs stochastiques gaussiens homogènes et non homogènes. La Recherche Aérospatiale, 1 (1989) 41-61. 